\title{
Diagnosis of Idiopathic Tracheal Stenosis and Treatment With Papillotome Electrocautery and Balloon Bronchoplasty
}

\author{
William R Solly MSc RRT CPFT, Richard J O'Connell RPFT, Hans J Lee MD, \\ Daniel H Sterman MD, and Andrew R Haas MD PhD
}

\section{Introduction}

Tracheal stenosis has numerous possible etiologies, including iatrogenic trauma from an over-inflated endotracheal/tracheostomy tube cuff, malignancy, chronic inflammatory diseases (eg, sarcoidosis), and various collagen vascular diseases (eg, Wegener disease, relapsing polychondritis). If an underlying etiology cannot be identified, the condition is termed idiopathic tracheal stenosis.

First reported by Brandenburg in 1972, idiopathic tracheal stenosis is a condition of the upper trachea and occurs overwhelmingly in females, in their third to fifth decade of life. ${ }^{1,2}$ Patients with idiopathic tracheal stenosis typically present with symptoms of dyspnea, limited exercise tolerance, stridor, cough, and wheezing. ${ }^{1-3}$ Diagnosis of idiopathic tracheal stenosis is usually made after other causes have been ruled out, and may be concurrently made with taking of medical history and physical examination. The diagnosis is confirmed with chest radiograph, computed tomogram, pulmonary-function tests, and/or bronchoscopy. It has been suggested that gastroesophageal reflux disease can cause idiopathic tracheal stenosis, and some authors have reported improvement in idiopathic tra-

\footnotetext{
Mr Solly, Dr Sterman, and Dr Haas are affiliated with the Section of Interventional Pulmonary and Thoracic Oncology; and Mr O'Connell is affiliated with Pulmonary Diagnostic Services/Respiratory Care, Division of Pulmonary, Allergy, and Critical Care Medicine, Hospital of the University of Pennsylvania, Philadelphia, Pennsylvania. Dr Lee is affiliated with the Section of Interventional Pulmonary, Division of Pulmonary and Critical Care Medicine, Virginia Commonwealth University Medical Center, Richmond, Virginia.
}

Dr Sterman has disclosed a relationship with Olympus Medical. The other authors have disclosed no conflicts of interest.

Correspondence: William R Solly MSc RRT CPFT, Pulmonary, Allergy, and Critical Care, Hospital of the University of Pennsylvania, Penn Lung Center, 1st Floor, West Pavilion, 3400 Civic Center Boulevard, Philadelphia PA 18974. E-mail: randy.solly@uphs.upenn.edu.

DOI: $10.4187 /$ respcare.01143 cheal stenosis with treatment for gastroesophageal reflux disease. ${ }^{4-6}$

An accurate diagnosis and proper treatment plan are required to restore an adequate patent airway in a patient with idiopathic tracheal stenosis. The definitive treatment routinely entails single-stage laryngotracheal resection. Successful airway restoration and voice preservation occurs in more than $90 \%$ of patients; however, post-procedural tracheal scarring and stenosis recurrence are potential complications. ${ }^{7}$ Factors determining surgical resection versus endoscopic management include the patient's age; location, size, and appearance of the stenosis; comorbidities; and previous interventions. ${ }^{8}$

\section{Case Summary}

A 25-year-old female non-smoker presented with insidious dyspnea progression associated with worsening exercise intolerance over a 9-month period. She believed her symptoms were due to physical deconditioning. Initial examination revealed a well nourished female, height $1.75 \mathrm{~cm}$, weight $103 \mathrm{~kg}$, body mass index $33.7 \mathrm{~kg} / \mathrm{m}^{2}$, blood pressure $117 / 75 \mathrm{~mm} \mathrm{Hg}$, heart rate 68 beats/min, temperature $36.8^{\circ} \mathrm{C}$, respiratory rate 16 breaths/min, and $\mathrm{S}_{\mathrm{pO}_{2}} 99 \%$ on room air. Her physical examination was notable for audible breathing during quiet inspiration and expiration, and stridor during rapid forced inspiration. Her medical, social, and family histories included no noteworthy pulmonary disease or occupational or environmental exposure.

Her pre-bronchodilator spirometry values were forced vital capacity (FVC) $3.68 \mathrm{~L}$ ( $81 \%$ of predicted), $\mathrm{FEV}_{1} 2.94 \mathrm{~L}$ (76\% of predicted), $\mathrm{FEV}_{1} / \mathrm{FVC} 80 \%$, peak expiratory flow $4.25 \mathrm{~L} / \mathrm{s}$ ( $54 \%$ of predicted), and there were no substantial changes after bronchodilator administration. The truncated inspiratory and expiratory flow-volume loops had the classic appearance of fixed upper-airway obstruction (Fig. 1A). Computed tomogram showed a narrowed trachea approximately $3 \mathrm{~cm}$ distal to the vocal cords, indicative of airway stenosis. She was referred to our hospital, where she had bronchoscopy, airway evaluation, and endoscopic stenosis management. 

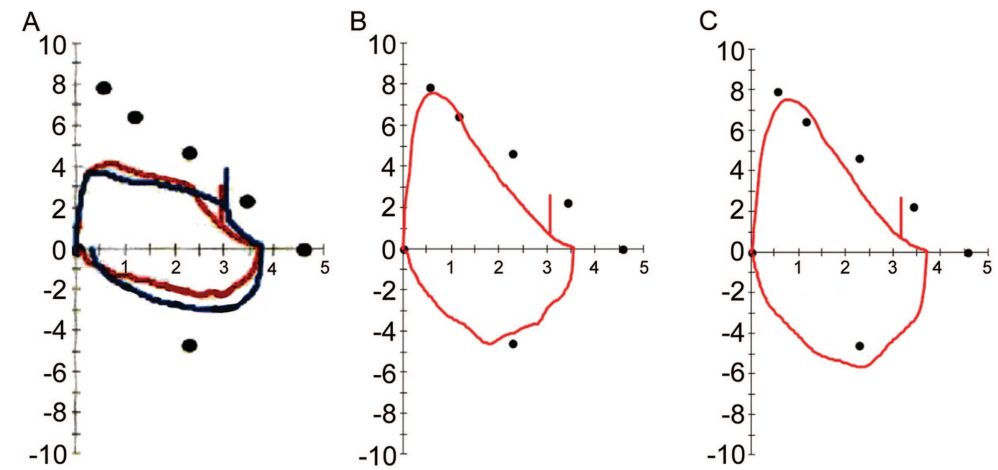

Fig. 1. Flow-volume loops. A: At baseline, before and after bronchodilator. B: Six weeks after papillotome electrocautery and balloon bronchoplasty. C: Fourteen weeks after the procedure.

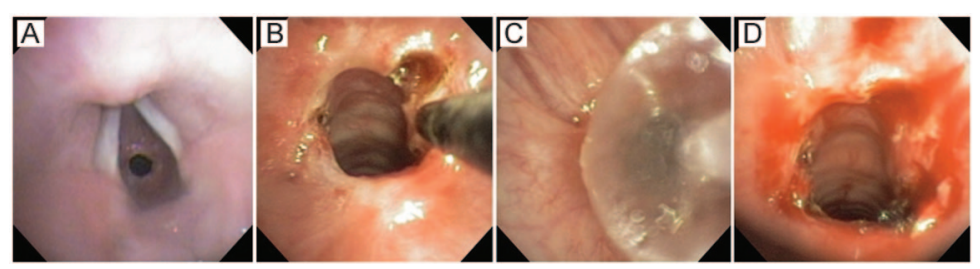

Fig. 2. A: Tracheal stenosis $3 \mathrm{~cm}$ distal to the vocal cords. B: During electrocautery with needle knife papillotome. C: During balloon bronchoplasty. D: After electrocautery and balloon bronchoplasty.

\section{Bronchoscopy and Papillotome Electrocautery With Balloon Bronchoplasty}

Bronchoscopy (BF-3C160 small-diameter bronchoscope, Olympus, Tokyo, Japan), with the patient conscious and in the upright position, revealed a circumferential fibrotic web-like subglottic stenosis, $3 \mathrm{~mm}$ long, inner diameter $5 \mathrm{~mm}, 3 \mathrm{~cm}$ below the vocal cords, just distal to the cricoid cartilage (Fig. 2A). The bronchoscope easily passed through the stenosis, and the distal trachea and main bronchi were normal. Then we put her under general anesthesia, placed a laryngeal mask airway, and mechanically ventilated with continuous spontaneous ventilation, pressure support of $12 \mathrm{~cm} \mathrm{H}_{2} \mathrm{O}$, and PEEP of $5 \mathrm{~cm} \mathrm{H}_{2} \mathrm{O}$ throughout the papillotome electrocautery and balloon bronchoplasty procedure. A rigid bronchoscope was readily available if needed.

With a 2.0-mm electrocautery needle knife papillotome (Cook Medical, Winston-Salem, North Carolina), through the working channel of a standard adult therapeutic bronchoscope (1T-180, Olympus, Tokyo, Japan), fine radial incisions were made at the 2:00, 5:00, 7:00, 9:00, and 10:00 o'clock positions on the stenosis (see Fig. 2B). There was no bleeding or noteworthy collateral injury to the adjacent normal mucosa. The stenosis was then serially dilated with 6/7/8-mm, 8/9/10-mm, and 10/11/12-mm pulmonary balloon dilators (CRE, Boston Scientific, Natick, Massachusetts), which dramatically improved subglottic patency (see Fig. 2C and 2D). There was no evidence of induced edema or tearing of adjacent mucosa.

Following the final balloon dilation, the laryngeal mask airway was removed, and the patient was breathing spontaneously in the operating room. In the recovery area she noted immediate improvement in her breathing and resolution of stridor. She was discharged with a prescription for pantoprazole (40 mg twice daily). At her 6-weeks follow-up visit, she was asymptomatic; her spirometry values were FVC $3.56 \mathrm{~L}$ (78\% of predicted), $\mathrm{FEV}_{1} 3.07 \mathrm{~L}(80 \%$ of predicted), $\mathrm{FEV}_{1} / \mathrm{FVC} \% 80 \%$ of predicted, and peak expiratory flow $7.75 \mathrm{~L} / \mathrm{s}$ (98\% of predicted). At her 14weeks follow-up visit she remained stable, and her spirometry values were FVC $3.72 \mathrm{~L}$ ( $82 \%$ of predicted), $\mathrm{FEV}_{1} 3.17 \mathrm{~L}$ (82\% of predicted), $\mathrm{FEV}_{1} / \mathrm{FVC}^{2} 85 \%$ of predicted, and peak expiratory flow $7.97 \mathrm{~L} / \mathrm{s}(101 \%$ of predicted). Her pre-procedure inspiratory and expiratory flowvolume loop truncation had resolved (Fig. 1B and 1C).

\section{Discussion}

\section{Spirometry}

The usefulness of pulmonary function testing in diagnosing respiratory disease is well established. Of special interest is when simple pulmonary function testing aids in the diagnosis of a relatively uncommon pathology such as an upper-airway obstruction (ie, in the pharynx, larynx, or 


\section{Diagnosis and Treatment of Idiopathic Tracheal Stenosis}

extrathoracic portion of the trachea). ${ }^{9}$ To fully appreciate the spirometry results from our patient, a brief overview of flow-volume loop maneuvers is warranted.

During normal inspiration, the negative intrathoracic pressure created by diaphragm contraction causes the intrathoracic airway diameter to expand as the lungs expand. In contrast, the extrathoracic airway constricts because the increase in air flow decreases the intraluminal pressure, which makes the extrathoracic airway pressure less than atmospheric pressure, due to the Bernoulli effect. The opposite phenomenon occurs during expiration: the intrathoracic airway diameter shrinks and the extrathoracic airway diameter expands. ${ }^{9,10}$ While these airway and flow dynamics are benign and clinically unimportant in healthy individuals, when the airway diameter is reduced due to a stenosis, the air-flow limitation can cause symptoms, depending on the degree of narrowing. ${ }^{11} \mathrm{Al}$-Bazzaz et al ${ }^{12}$ and Geffin et $\mathrm{al}^{13}$ reported that a stenosis that reduces upper-airway diameter to $8 \mathrm{~mm}$ produces symptoms during exercise, and a stenosis that reduces the airway to $5 \mathrm{~mm}$ causes at-rest inspiratory obstruction and stridor. Such an obstruction pattern is readily identifiable on a screening-spirometry flow-volume loop and can be the first indication of the stenosis location and severity, and can help predict the outcomes of intervention. ${ }^{9} 14,15$

The typical appearance of airway obstruction on the flow-volume loop is concavity, due to reduced flow, versus the reduced volume seen in restrictive disease. In central airway obstruction the flow-volume loop can take 3 different shapes, depending on the location of the pathology: fixed airway obstruction, variable extrathoracic obstruction, and variable intrathoracic obstruction. ${ }^{9,10,14-16}$

Fixed airway obstruction can be intrathoracic or extrathoracic. Regardless of the location, the impedance to flow does not vary with the dynamic fluctuations of airway size during the respiratory cycle; the flow limitation occurs during both inspiration and expiration, and the flow impedance is dramatically accentuated during an FVC maneuver. The resultant flow-volume loop is flattened on both the inspiratory and expiratory limbs, as seen in our patient's initial flow-volume loop., ${ }^{9,15}$

Variable obstruction is characterized by changes in air flow depending on the location of the obstruction and the phase of the respiratory cycle. In variable extrathoracic obstruction the expiratory limb is relatively normal, since extrathoracic intratracheal pressure exceeds atmospheric pressure, thereby maintaining extrathoracic airway patency and allowing air to flow past the obstruction. During inspiration the acceleration of air flow decreases intraluminal pressure relative to atmospheric pressure, which narrows the extrathoracic airway, and the stenosis or other obstruction (eg, vocal cord paralysis, goiter, or laryngeal tumor) reduces inspiratory air flow, producing the classical flattening or blunting of the inspiratory limb..$^{9,15,16}$

In contrast, variable intrathoracic obstruction creates the opposite dynamic. During inspiration the airways enlarge as the lungs expand, and the inspiratory limb is normal. During expiration the thoracic pressure exceeds airway pressure, which narrows the intrathoracic airway, further reduces expiratory air flow, and flattens or blunts the expiratory limb. ${ }^{9}$

An aid in determining the site of an upper-airway obstruction is to compare the expiratory and inspiratory flow at $50 \%$ of the FVC (ie, $\mathrm{FEF}_{50}$ and $\mathrm{FIF}_{50}$ ). Healthy individuals have an $\mathrm{FEF}_{50} / \mathrm{FIF}_{50}$ of 1.0. A variable extrathoracic stenosis (which shows normal expiratory flow and reduced inspiratory flow) will cause an $\mathrm{FEF}_{50} / \mathrm{FIF}_{50}$ of $>1.0$. A variable intrathoracic obstruction (reduced expiratory flow and normal inspiratory flow) will cause an $\mathrm{FEF}_{50} / \mathrm{FIF}_{50}$ of $<1.0 . .^{9,14,16}$

\section{Stenosis Management}

There are several approaches to the management of tracheal stenosis. While physical examination, flow-volume studies, and computed tomography are critical in the diagnosis, the stenosis should be confirmed visually via bronchoscopy to determine the character of the stenosis. In our patient we used a small-diameter bronchoscope to minimize airway resistance and avoid further compromise of her respiratory status.

Since bronchoscopy is important in tracheal stenosis management, balloon dilation of the stenosis can be easily and safely performed at the same time. Balloon dilation is often the initial treatment of tracheal stenosis and can be performed under moderate sedation. There have been no reported deaths due to balloon dilation, and it is a rapid technique to improve a stenosis and symptoms. Approximately $47-71 \%$ of patients with non-malignant disease may be managed with balloon dilation alone. ${ }^{17}$ In our patient we conducted controlled stenosis release with carefully placed fine electrocautery incisions, rather than random and irregular tears that might cause an airway injury. In our experience, the precise incisions possible with a papillotome, compared to a standard electrocautery blade, limit tissue injury.

As in our patient, the flow-volume loop can be an important diagnostic tool in the evaluation of possible tracheal stenosis, and most stenoses can be initially managed bronchoscopically with long-term benefit. ${ }^{17,18}$ However, for stenoses that are complex, recur, or are associated with malacia, additional techniques (not used in our patient) such as airway stenting may be needed. Some tracheal stenoses require tracheotomy, Montgomery T-tube insertion, or surgical reconstruction or resection if bronchoscopic approaches are unsuccessful. 


\section{Diagnosis and Treatment of Idiopathic Tracheal Stenosis}

\section{Teaching Points}

- Idiopathic tracheal stenosis should be considered when other more common diagnoses such as asthma have been excluded.

- Accurate spirometry is crucial. While pulmonary function tests help detect and quantify an upper-airway obstruction, remember that the FVC maneuver and resultant flow-volume loop are effort-dependent. The appearance of inspiratory or expiratory obstruction can be caused by poor patient effort, and attention must be given to assuring maximum subject effort and repeatable results. The spirometry technician must be knowledgeable and properly trained in coaching patients to meet the American Thoracic Society/European Respiratory Society criteria.

- Bronchoscopy is an important tool in tracheal stenosis management, as it confirms the diagnosis and allows characterizing the stenosis, which guides treatment.

- Bronchoscopic tools such as balloon bronchoplasty and electrocautery incisions are safe and rapid treatments that can also be performed during diagnostic bronchoscopy and can limit the need for more invasive surgical procedures.

\section{REFERENCES}

1. Brandenburg JH. Idiopathic tracheal stenosis. Trans Am Acad Opthalmol Otolaryngol 1972;76(5):1402-1406.

2. Grillo HC, Mark EJ, Mathisen DJ, Wain JC. Idiopathic laryngotracheal stenosis and its management. Ann Thorac Surg 1993;56(1): 80-87.

3. Rossi C, Colombari F, Guembarowsky AL, Filho O, Thomson JC. Idiopathic tracheal stenosis. A report of four cases. J Bras Pneumol 2007;33(1):101-104.
4. Maronian NC, Azadeh H, Waugh P, Hillel A. Association of laryngopharyngeal reflux disease and subglottic stenosis. Ann Otol Rhinol Laryngol 2001;110(7):606-612.

5. Toohill RJ, Ulualp SO, Shaker R. Evaluation of gastroesophageal reflux in patients with laryngotracheal stenosis. Ann Otol Rhinol Laryngol 1998;107(12):1010-1014.

6. Terra RM, de Medeiros IL, Minamoto H, Nasi A, Pego-Fernandes PM, Jatene FB. Idiopathic tracheal stenosis: successful outcome with antigastroesophageal reflux disease therapy. Ann Thorac Surg 2008; 85(4):1438-1439.

7. Ahiku SK, Kuzcu A, Grillo HC, Wright CD, Wain JC, Lo B, et al. Idiopathic larygotracheal stenosis: effective definitive treatment with laryngotracheal resection. Thorac Cardiovasc Surg 2004;127(1):99107

8. Shapsay SM, Rahbar R, Healy GB. Mitomycin: effects on laryngeal and tracheal stenosis, benefits and complications. Ann Otol Rhinol Laryngol 2001;110(1):1-6.

9. Ruppel G. Manual of pulmonary function testing, 9th edition. St Louis: Mosby Elsevier; 2009:58-60.

10. Sundar KM, Kanner RE. Pulmonary function testing in diagnosing upper-airway obstruction. RT magazine; Oct/Nov 2000. http://www. rtmagazine.com/issues/articles/2000-10_11.asp. Accessed August 9, 2011.

11. Acres JC, Kryger MH, Clinical significance of pulmonary function tests: upper airway obstruction. Chest 1981;80(2): 207-211.

12. Al-Bazzaz F, Grillo H, Kazemi H, Responses to exercise in upper airway obstruction. Am Rev Respir Dis 1975;111(5):631-640.

13. Geffin B, Grillo H, Cooper JD, Pontoppidan H. Stenosis following tracheostomy for respiratory care. JAMA 1971;216(12):1984-1988.

14. Pellegrino R, Viegi G, Brusasco, V, Crapo RO, Burgos F, Casaburi $\mathrm{R}$, et al. Interpretative strategies for lung function tests. Eur Respir $\mathrm{J}$ 2005;26(5):948-968

15. Soto FJ, Guntupalli, KK. All that wheezes is not asthma: diagnosing the mimics. Emerg Med 2001;33(11):40-56.

16. Vlahakis NE, Patel AM, Maragos NE, Beck KC. Diagnosis of vocal cord dysfunction: the utility of spirometry and plethysmography. Chest 2002;122(6):2246-2249.

17. McArdle J, Gildea T, Mehta A. Balloon bronchoplasty its indication, benefits and complications. J Bronch 2005;12(2):123-127.

18. Tremblay A, Coulter T, Mehta A. Modification of a mucosal-sparing technique using electrocautery and balloon dilation in the endoscopic management of web-like benign airway stenosis. J Bronchol 2003; 10(4):268-271. 